ARTICLE

https://doi.org/10.1038/s41467-019-09788-5

\title{
Selective photoelectrochemical oxidation of glycerol to high value-added dihydroxyacetone
}

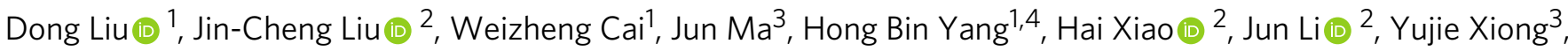
Yanqiang Huang ${ }^{5} \&$ Bin Liu $^{1}$

It is highly profitable to transform glycerol - the main by-product from biodiesel production to high value-added chemicals. In this work, we develop a photoelectrochemical system based on nanoporous $\mathrm{BiVO}_{4}$ for selective oxidation of glycerol to 1,3-dihydroxyacetone - one of the most valuable derivatives of glycerol. Under AM $1.5 \mathrm{G}$ front illumination $\left(100 \mathrm{~mW} \mathrm{~cm}^{-2}\right)$ in an acidic medium $(\mathrm{pH}=2)$ without adscititious oxidant, the nanoporous $\mathrm{BiVO}_{4}$ photoanode achieves a glycerol oxidation photocurrent density of $3.7 \mathrm{~mA} \mathrm{~cm}^{-2}$ at a potential of $1.2 \mathrm{~V}$ versus RHE with 51\% 1,3-dihydroxyacetone selectivity, equivalent to a production rate of $200 \mathrm{mmol}$ of 1,3-dihydroxyacetone per $\mathrm{m}^{2}$ of illumination area in one hour.

\footnotetext{
${ }^{1}$ School of Chemical and Biomedical Engineering, Nanyang Technological University, 62 Nanyang Drive, Singapore 637459, Singapore. ${ }^{2}$ Department of Chemistry and Key Laboratory of Organic Optoelectronics \& Molecular Engineering of Ministry of Education, Tsinghua University, Beijing 100084, China. ${ }^{3}$ Hefei National Laboratory for Physical Sciences at the Microscale, Collaborative Innovation Center of Chemistry for Energy Materials (iChEM), School of Chemistry and Materials Science, University of Science and Technology of China, Hefei 230026, China. ${ }^{4}$ Institute for Materials Science and Devices, Suzhou University of Science and Technology, Suzhou 215009, China. ${ }^{5}$ State Key Laboratory of Catalysis, Dalian Institute of Chemical Physics, Chinese Academy of Sciences, Dalian 116023, China. Correspondence and requests for materials should be addressed to H.X. (email: haixiao@tsinghua.edu.cn) or to Y.X. (email: yjxiong@ustc.edu.cn) or to B.L. (email: liubin@ntu.edu.sg)
} 
ncreasing global energy demand and environmental concern drive the development of fuels and building-block chemicals production from renewable feedstocks and green energy sources $^{1-6}$. Biodiesel manufactured from vegetable oils and animal fats has become increasingly attractive because of its environmental benefits $^{7-10}$, which is now produced at million tonnes of oil equivalent per year. Production of 100 pounds of biodiesel brings out 10 pounds of glycerol as a by-product. With increase in global biodiesel consumption, efficient utilization of glycerol becomes an urgent and highly profitable market ${ }^{11-17}$. Glycerol has many oxidation derivatives, among which, 1,3-dihydroxyacetone (DHA) is most valuable. DHA is widely used in cosmetic, pharmaceutical, fine chemical, and food industries, which costs US $\$ 150$ per $\mathrm{kg}$ while the price for crude and refined glycerol is only US $\$ 0.11$ and US $\$ 0.66$ per $\mathrm{kg}$, respectively ${ }^{18,19}$. Commercial DHA is mainly produced via a microbial fermentation process, which is complex, time-consuming, and subject to substrate inhibition ${ }^{15,20}$. Besides, selective catalytic conversion of glycerol to DHA could be achieved by thermal oxidation or electrochemical oxidation on noble metal catalysts (e.g., Pt, Pd, $\mathrm{Ag}$, and $\mathrm{Au}$ ) with adscititious oxidants ${ }^{21-28}$, which are not only cost-intensive, but also produce DHA at very low production rates with poor DHA selectivities.

Photoelectrochemical oxidation provides another promising approach, which combines both renewable feedstock and green energy source together to make building-block chemical and clean fuel at the same time. In this study, we demonstrate that photoelectrochemical oxidation of glycerol to DHA could be achieved with high selectivity at ambient temperature and pressure on porous $\mathrm{BiVO}_{4}$. At $\mathrm{pH}=2$, under $\mathrm{AM} 1.5 \mathrm{G}$ front illumination $\left(100 \mathrm{~mW} \mathrm{~cm}^{-2}\right)$, the porous $\mathrm{BiVO}_{4}$ photoanode is able to produce about $200 \mathrm{mmol}$ of DHA per $\mathrm{m}^{2}$ of illumination area in $1 \mathrm{~h}$ at a potential of $1.2 \mathrm{~V}$ vs. RHE without the requirement of adscititious oxidant. Based on isotope labeling experiments and density functional theory (DFT) calculations, we deduce a possible reaction mechanism of selective photoelectrochemical oxidation of glycerol to $\mathrm{DHA}$ on $\mathrm{BiVO}_{4}$.

\section{Results}

Synthesis and structural characterization of $\mathrm{BiVO}_{4}$ photoanode. $\mathrm{BiVO}_{4}$ photoanode was prepared by reacting $\mathrm{BiOI}$ nanoflake arrays that were electrochemically grown on fluorinedoped tin dioxide (FTO) glass with vanadyl acetylacetonate at elevated temperatures. Supplementary Fig. 1 shows the typical scanning electron microscopy (SEM) image of the as-prepared $\mathrm{BiVO}_{4}$ photoanode, which displays porous, interconnected nanoflake arrays. High-resolution transmission electron microscopy (HRTEM) image shows that the main exposed facet of the $\mathrm{BiVO}_{4}$ nanoflake is (112). The $\mathrm{BiVO}_{4}$ nanoarrays are phase-pure with monoclinic scheelite structure (Supplementary Fig. 2). The bandgap of the $\mathrm{BiVO}_{4}$ nanoarrays was measured to be $\sim 2.5 \mathrm{eV}$ (Supplementary Fig. 3).

Photoelectrochemical performance of $\mathrm{BiVO}_{4}$ photoanode. Photoelectrochemical (PEC) performance of $\mathrm{BiVO}_{4}$ photoanode was first evaluated in $0.5 \mathrm{M} \mathrm{Na}_{2} \mathrm{SO}_{4}$ electrolyte with $\mathrm{pH}$ adjusted from 2 to 12 under one sun illumination (AM $1.5 \mathrm{G}, 100 \mathrm{~mW} \mathrm{~cm}^{-2}$ ). Figure 1 shows the current density-potential profiles under dark and illumination conditions. Without the addition of glycerol in the reaction medium, the photocurrent density resulting from water oxidation via front illumination is nearly identical, reaching $\sim 1 \mathrm{~mA}$ $\mathrm{cm}^{-2}$ at $1.23 \mathrm{~V}$ vs. RHE independent of $\mathrm{pH}$. When glycerol was introduced, the photocurrent density dramatically increased, accompanying with a clear onset shift towards lower potentials, indicating easier oxidation of glycerol than water. The photocurrent density approaches $4 \mathrm{~mA} \mathrm{~cm}^{-2}$ at $1.23 \mathrm{~V}$ vs. $\mathrm{RHE}$ at $\mathrm{pH}=2$. Increase in $\mathrm{pH}$ greatly decreases photocurrent and at the same time increases the onset potential, suggesting that proton could directly influence the catalytic oxidation reaction. Further increasing $\mathrm{pH}$ to more than 10 caused photocorrosion of $\mathrm{BiVO}_{4}$ (Supplementary Fig. 4).

Figure $1 \mathrm{~d}-\mathrm{f}$ and Supplementary Fig. 5 show the chopped photocurrent profiles recorded at 1.0 and $0.6 \mathrm{~V}$ vs. RHE, respectively in an $\mathrm{H}$-type cell. In the absence of glycerol, each on-off cycle of photocurrent produces a transient spike, which is induced by the diffusion and accumulation of photogenerated holes at the surface of $\mathrm{BiVO}_{4}$ due to the slow kinetics of water oxidation reaction (Supplementary Fig. 6 and Supplementary Note 1$)^{29}$. The stable photocurrent density reaches 0.1 and $1.0 \mathrm{~mA} \mathrm{~cm}^{-2}$ at 0.6 and $1.0 \mathrm{~V}$ vs. RHE, respectively at $\mathrm{pH}=2$. Adding $0.1 \mathrm{M}$ of glycerol not only dramatically increases the photocurrent density to 1.8 and $3.4 \mathrm{~mA} \mathrm{~cm}^{-2}$ at 0.6 and $1.0 \mathrm{~V}$ vs. RHE, but also at the same time greatly reduces the photocurrent spike. This comparison suggests faster reaction kinetics of glycerol oxidation as compared to water oxidation. Additionally, increase in reaction $\mathrm{pH}$ greatly reduces the photocurrent density, which can be attributed to the better glycerol adsorption on $\mathrm{BiVO}_{4}$ at lower $\mathrm{pH}$ (Supplementary Fig. 7 and Supplementary Note 2). Enhanced adsorption of glycerol on $\mathrm{BiVO}_{4}$ at lower $\mathrm{pH}$ shall result in easier transfer of photogenerated holes for further oxidation reactions, which therefore diminishes the chopped photocurrent spikes.

To gain information on the charge and mass transfer processes, electrochemical impedance spectroscopy (EIS) studies were performed under different applied biases with light illumination. An equivalent circuit based on charge carrier trapping mechanism was employed to analyze the EIS data (Fig. 2c) ${ }^{30,31}$. The equivalent circuit includes a space-charge capacitance, $C_{\mathrm{sc}}$ and a surface state capacitance, $\mathrm{CPE}_{\varphi}$. $R_{\Omega}$ is the series resistance, $R_{\mathrm{t}}$ is the resistance that represents the trapping of holes in the surface states, and $R_{\mathrm{s}}$ is the charge and mass transfer resistance from surface states to solution. For the EIS spectra collected without glycerol, as shown in the Bode (Fig. 2a) and Nyquist plots (Supplementary Fig. 8a), the frequency peak decreases and shifts to higher frequency with increasing bias, leading to decreased faradaic resistance and improved surface reaction rate. After adding glycerol, the frequency peak shifts to higher frequency, but the impedance semicircle becomes larger with increasing bias (Fig. $2 \mathrm{~b}$ and Supplementary Fig. $8 \mathrm{~b}$ ). The increased $R_{\mathrm{s}}$ in PEC glycerol oxidation with increasing bias could be attributed to the poor desorption of the oxidation products ${ }^{32}$. We further calculated the total resistance, $R_{\text {total }}$ via $R_{\text {total }}=R_{\Omega}+R_{\mathrm{t}}+R_{\mathrm{s}}$ (Supplementary Fig. $8 \mathrm{c}$ and Supplementary Note 3), and compared with the resistance estimated from the current density-potential profile $\left(R_{\text {total }}=\mathrm{d} V \mathrm{~d} J^{-1}\right)$ as shown in Fig. $2 \mathrm{~d}$. At potentials prior to $\mathrm{H}_{2} \mathrm{O}$ oxidation onset, $R_{\text {total }}$ is found to be orders of magnitude higher for PEC water oxidation as compared to that for PEC glycerol oxidation.

To probe the charge carrier dynamics, we performed transient open-circuit voltage decay (OCVD) measurements. Figure 3a compares the OCVD profiles of the $\mathrm{BiVO}_{4}$ photoanode measured in $0.5 \mathrm{M} \mathrm{Na}_{2} \mathrm{SO}_{4}$ aqueous solution at various $\mathrm{pH}$, from which, we were able to estimate the lifetimes of photogenerated charge carriers (Fig. 3b). It is found that decrease in reaction $\mathrm{pH}$ slightly increases recombination rate of photogenerated electron-hole pairs. The photoresponse spectra of the porous $\mathrm{BiVO}_{4}$ nanoarray photoanode were further assessed as shown in Fig. $3 c$, d, which resemble well with the light absorption spectrum of $\mathrm{BiVO}_{4}$ (Supplementary Fig. 3). In the absence of glycerol at $\mathrm{pH}=2$, the incident photon-to-current conversion efficiency (IPCE) approaches $\sim 20 \%$ at $400 \mathrm{~nm}$ and $1.23 \mathrm{~V}$ vs. RHE. The addition 

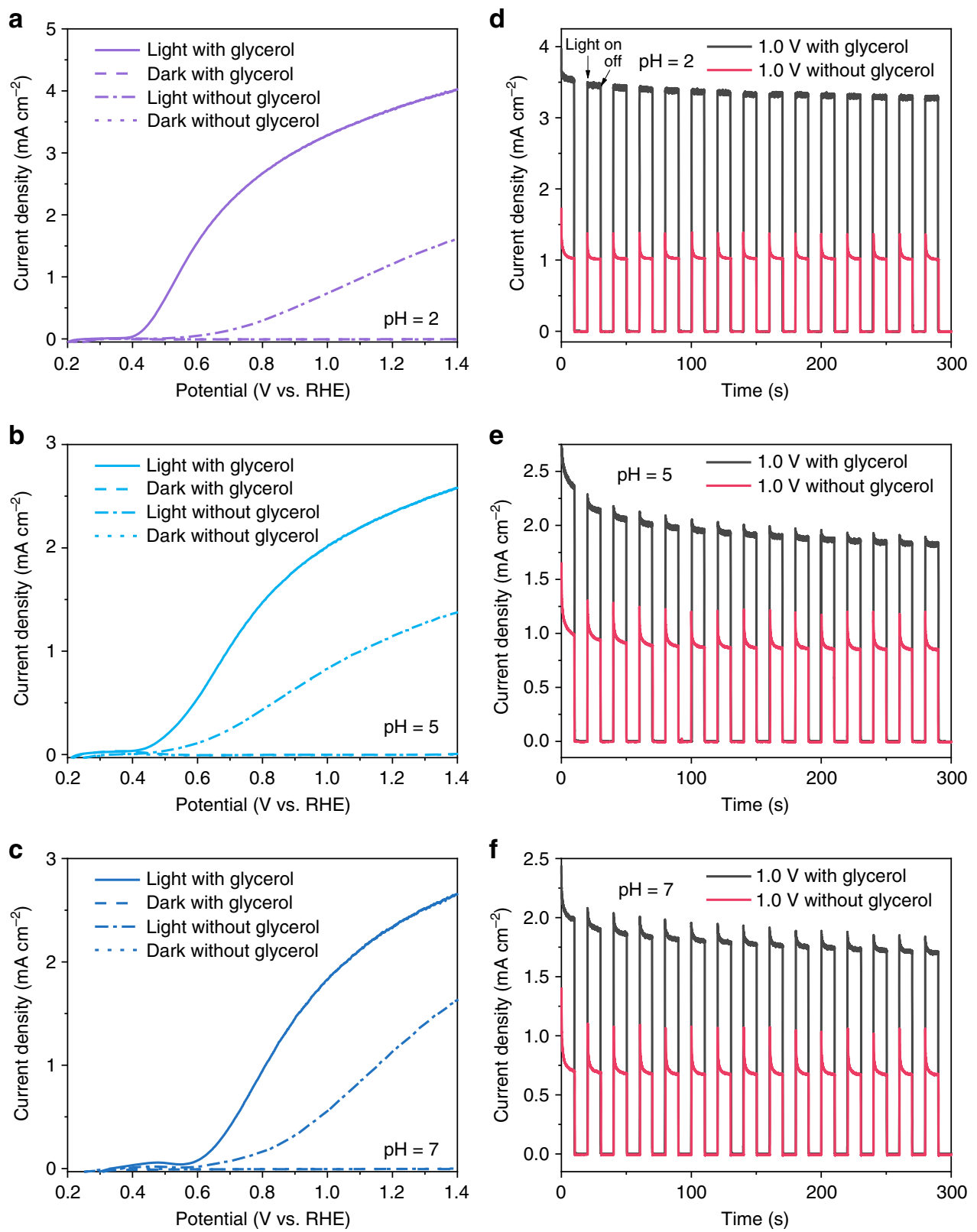

Fig. 1 Photoelectrochemical performances. a-c Current density-potential profiles of $\mathrm{BiVO}_{4}$ photoanode measured in $0.5 \mathrm{M} \mathrm{Na} 2 \mathrm{SO}_{4}$ at various pH in an $\mathrm{H}$ type cell under dark and $\mathrm{AM} 1.5 \mathrm{G}, 100 \mathrm{~mW} \mathrm{~cm}{ }^{-2}$ illumination. d-f Chopped photocurrent density-time profiles of $\mathrm{BiVO}_{4}$ photoanode at $1.0 \mathrm{~V}$ vs. RHE in $0.5 \mathrm{M} \mathrm{Na}_{2} \mathrm{SO}_{4}$ at various $\mathrm{pH}$ with and without glycerol. Source data are provided as a Source Data file

of glycerol significantly increases the IPCE across the entire light absorption region of $\mathrm{BiVO}_{4}$, reaching $80 \%$ at $400 \mathrm{~nm}$ and $1.23 \mathrm{~V}$ vs. RHE, which is consistent with the change in photocurrent density. Furthermore, increase in reaction $\mathrm{pH}$ decreases IPCE (Fig. 3d) as a result of impaired binding of glycerol on $\mathrm{BiVO}_{4}$ as discussed previously.

Selective photoelectrochemical oxidation of glycerol. The photoelectrochemical stability of the porous $\mathrm{BiVO}_{4}$ nanoarray photoanode was assessed by measuring photocurrent as a function of reaction time at various applied potentials. As shown in Supplementary Fig. 9, the $\mathrm{BiVO}_{4}$ photoanode exhibits excellent long-time stability against photocorrosion at $\mathrm{pH}=2$ with stable photocurrent densities. Following, the photooxidation of glycerol was carried out at $0.8-1.2 \mathrm{~V}$ vs. RHE to analyze the oxidation products and faradaic efficiency (Supplementary
Fig. 10). The products consist of formic acid, glyceric acid, DHA, and trace amount of glycolic acid. Figure $4 \mathrm{a}$ and Supplementary Fig. 11 show the production rate and selectivity of oxidation products at various $\mathrm{pH}$. With increase in applied potential, the production rate of the oxidation products also increases. At $\mathrm{pH}=2$, DHA becomes the preferred oxidization product with selectivity and overall faradaic efficiency approaching $~ 50 \%$ in the liquid products and $\sim 30 \%$, respectively, independent of applied potential. At a potential of $1.2 \mathrm{~V}$ vs. RHE, the production rate of DHA reaches as high as about $200 \mathrm{mmol}$ per $\mathrm{m}^{2}$ of illumination area in $1 \mathrm{~h}$, and the total carbon balance is about $91.3 \%$, in which the glycerol-to-DHA conversion selectivity is 63.6\% (Supplementary Table 1). $\mathrm{CO}_{2}$ and $\mathrm{CO}$ could also be detected from further oxidation of liquid products together with $\mathrm{H}_{2} \mathrm{O}_{2}$ and $\mathrm{O}_{2}$ from water oxidation. Total charge-to-chemical balance is about $90.21 \%$. Besides DHA, glyceric acid was also produced as a by-product, resulting in the overall selectivity of 

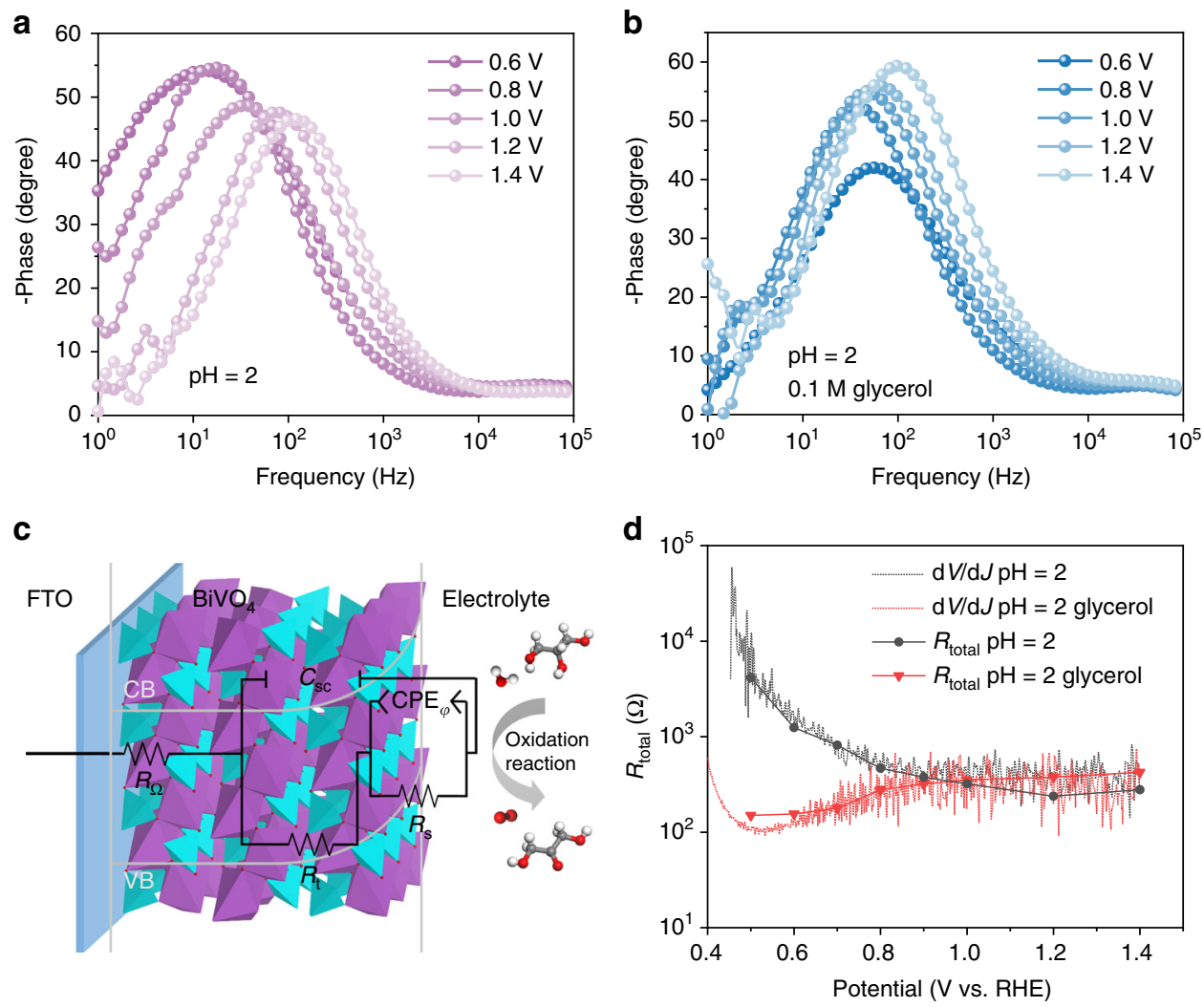

Fig. 2 Charge and mass transfer processes. a, b The Bode plots measured at different potentials under AM $1.5 \mathrm{G}, 100 \mathrm{~mW} \mathrm{~cm}^{-2}$ illumination without and with the presence of glycerol. c Equivalent circuit used to conceptualize the $\mathrm{BiVO}_{4}$ photoanode in $\mathrm{PEC}$ reactions. $\mathbf{d} R_{\text {total }}$ calculated from $\mathrm{dV} \mathrm{d} J^{-1}$ of current density-potential profiles and from EIS data with and without glycerol under illumination
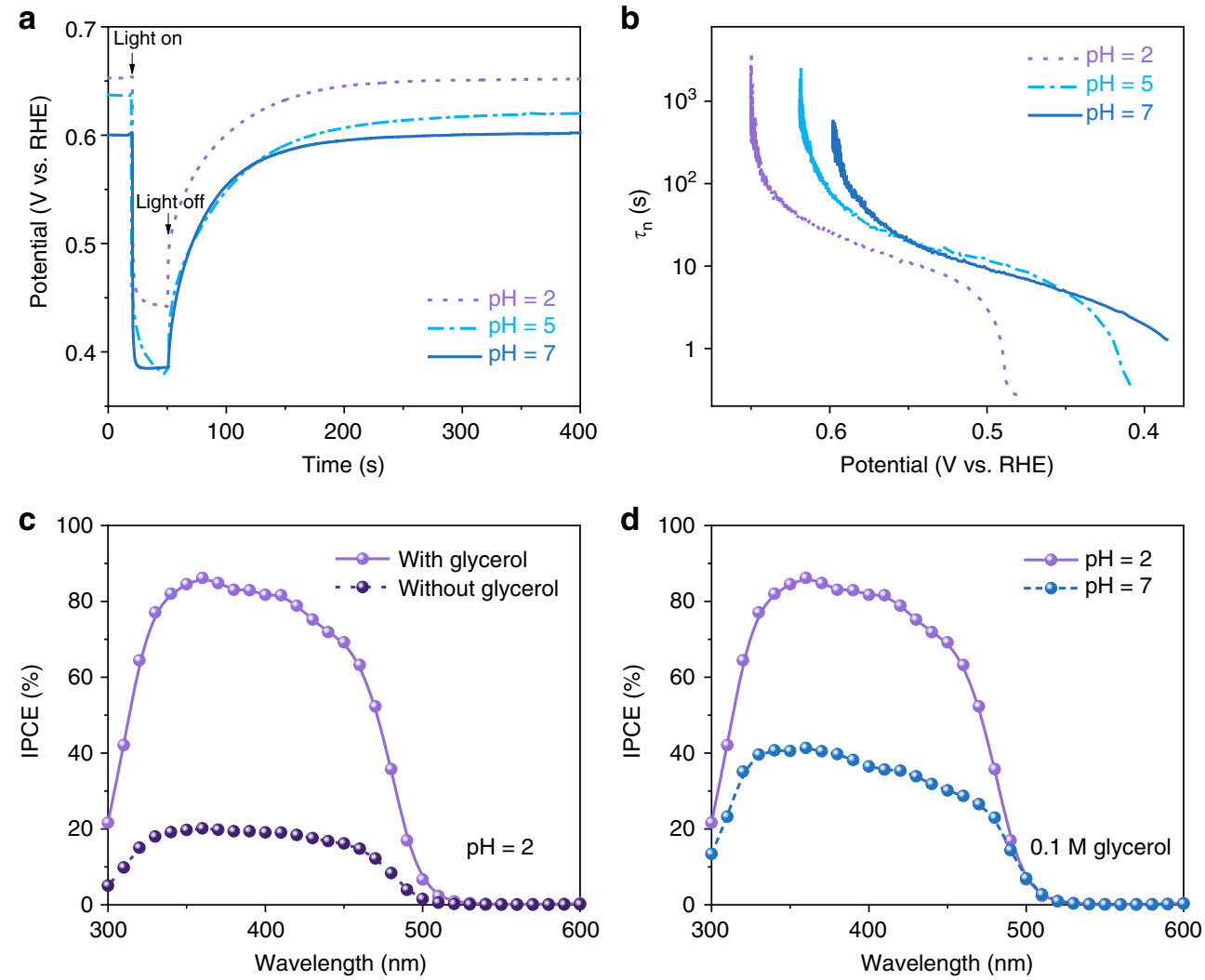

Fig. 3 Charge carrier dynamics. $\mathbf{a}, \mathbf{b}$ Transient OCVD profiles and lifetimes of photogenerated charge carriers of porous $\mathrm{BiVO}_{4}$ nanoarray photoanode in $0.5 \mathrm{M} \mathrm{Na}_{2} \mathrm{SO}_{4}$ at various $\mathrm{pH}$. c, $\mathbf{d}$ Incident photon-to-current conversion efficiency spectra of porous $\mathrm{BiVO}_{4}$ nanoarray photoanode 


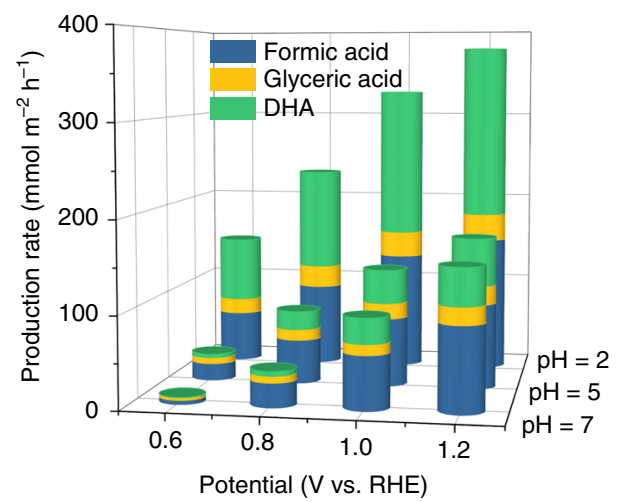

C

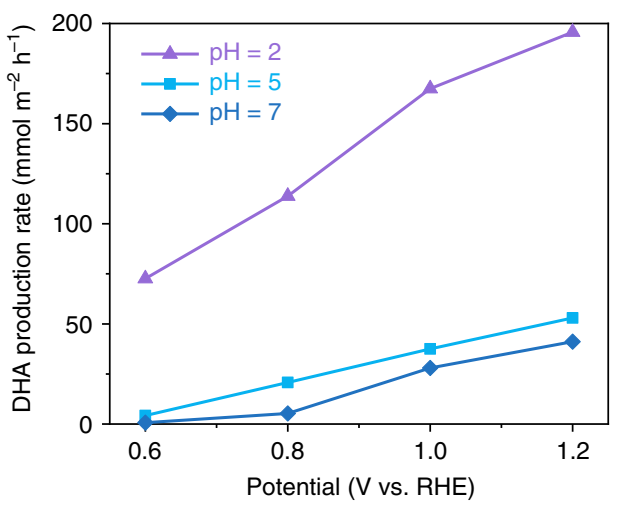

e

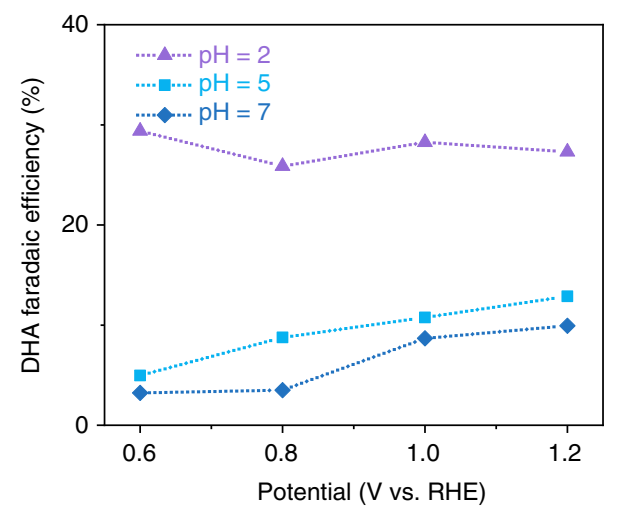

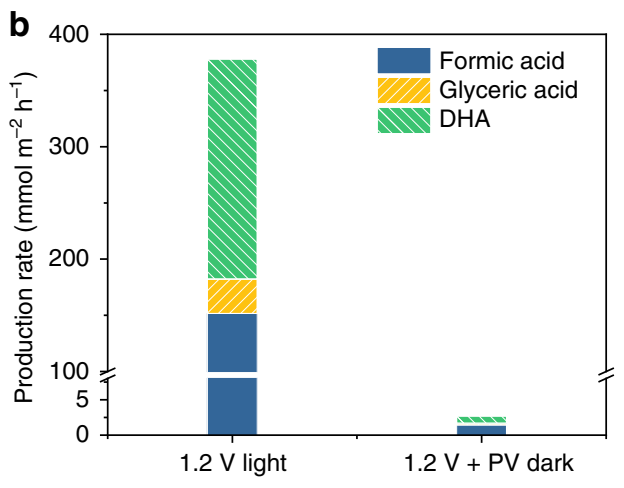

d
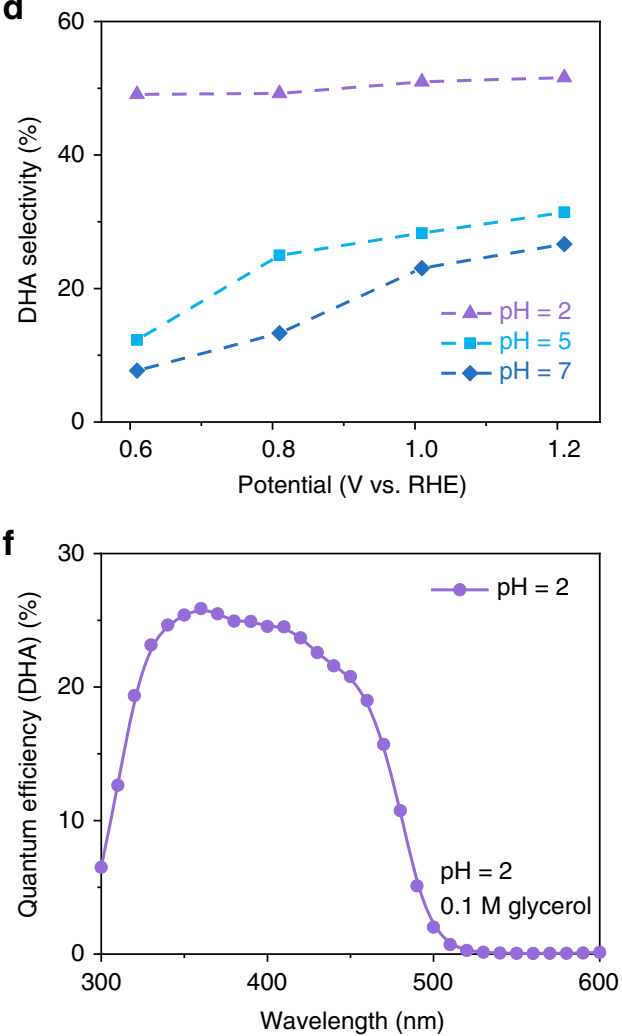

Fig. 4 Selective photoelectrochemical oxidation of glycerol. a Photoelectrocatalytic production rate of oxidation products on BiVO 4 photoanode at reaction $\mathrm{pH}$ of 2,5 , and 7 , respectively in $1 \mathrm{~h}$. $\mathbf{b}$ Production rate of oxidation products at $\mathrm{pH}=2$ and $1.2 \mathrm{~V}$ vs. RHE under illumination or $1.375 \mathrm{~V}$ vs. RHE in dark, respectively c-e Production rate, selectivity, and faradaic efficiency of DHA produced in an $\mathrm{H}$-type cell at $\mathrm{pH}=2$, 5 , and 7 . $\mathbf{f}$ Incident photon-to-DHA conversion efficiency spectrum acquired at $\mathrm{pH}=2$ and $1.23 \mathrm{~V}$ vs. $\mathrm{RHE}$

high value-added glycerol derivatives (DHA + glyceric acid) approaching $\sim 60 \%$. Increase in $\mathrm{pH}$ decreases not only the photocurrent, but also the selectivity towards high value-added oxidation products (Fig. $4 \mathrm{c}-\mathrm{e}$ ). At $\mathrm{pH}=12$, glycerol was almost completely oxidized to formic acid with a faradaic efficiency close to 99\% (Supplementary Fig. 12). Both photocurrent and production rate of DHA increase notably with increase of glycerol concentration (Supplementary Fig. 13). Without light illumination, glycerol could only be oxidized to $\mathrm{DHA}$ by $\mathrm{BiVO}_{4}$ at very low efficiency after applying additional bias (Fig. $4 \mathrm{~b}$ and Supplementary Fig. 14). Porous $\mathrm{BiVO}_{4}$ nanoarray photoanode could produce DHA with higher rate and selectivity than $\mathrm{BiVO}_{4}$ film photoanode as porous nanoarrays possess larger surface area and more abundant active sites (Supplementary Fig. 15 and Supplementary Note 4 ). In addition, porous $\mathrm{BiVO}_{4}$ nanoarrays also showed advantages in selective glycerol oxidation as compared to other semiconductor nanomaterials (Supplementary Fig. 16 and Supplementary Note 5).

Reaction mechanism. To probe the reaction mechanism, we conducted isotope-labeling experiments and electron spin resonance (ESR) measurements. Supplementary Fig. 17 displays the liquid chromatography mass spectrometry (LC-MS) results of photoelectrochemical glycerol oxidation in oxygen isotope labeled electrolyte with $\mathrm{H}_{2} \mathrm{O}$ containing $10 \% \mathrm{H}_{2}{ }^{18} \mathrm{O}$. The results show that ${ }^{18} \mathrm{O}$ can be detected in the target product DHA in the form of $\mathrm{C}_{3} \mathrm{H}_{6} \mathrm{O}_{2}{ }^{18} \mathrm{ONa}$ (Supplementary Table 2 and Supplementary Note 6), indicating that the isotope oxygen in DHA comes from water in the electrolyte. It can thus be speculated that oxidation of $\beta$-hydroxy to carbonyl group during photoelectrochemical glycerol oxidation to DHA would be accomplished by an addition 
and substitution reaction. The adsorbed glycerol on $\mathrm{BiVO}_{4}$ photoanode would be oxidized into radicals by photogenerated holes (Supplementary Tables 3, 4 and Supplementary Notes 7, 8) 33-35. Room temperature ESR spectra indicate that after adding glycerol in this system, instead of hydroxyl radicals, glycerol radicals could be detected (Supplementary Fig. 18 and Supplementary Note 9). Driven by the energetic holes, the glycerol radicals could react with water from electrolyte to form unstable gem-diol intermediates, followed by dehydration to produce the carbonyl group, during which the isotope oxygen might be left in the products ${ }^{35,36}$. As a conclusion, we infer that the oxidation of glycerol to DHA could occur via the following steps as shown in Supplementary Fig. 19 and Supplementary Note 10, including adsorption of glycerol on $\mathrm{BiVO}_{4}$ photoanode, oxidation of adsorbed glycerol to radicals by photogenerated holes, reaction with water, and dehydration. Glyceric acid and formic acid could also be formed via this similar pathway, resulted from the further oxidation of glyceraldehyde.

As shown in Supplementary Fig. 7, glycerol could be better adsorbed on $\mathrm{BiVO}_{4}$ at lower $\mathrm{pH}$, which shall result in an easier charge transfer and enhanced catalysis to photoelectrochemically convert glycerol into glycerol derivatives when decreasing reaction $\mathrm{pH}$. Besides, acidic environment can also suppress the reaction towards acid products, which might further reduce the consumption of DHA as well as suppress the production of formic acid ${ }^{37}$. The adsorption of glycerol on $\mathrm{BiVO}_{4}$ and the reaction pathways of the glycerol oxidation were verified by DFT calculations on the (112) facets of $\mathrm{BiVO}_{4}$-the main exposed crystal facets of the $\mathrm{BiVO}_{4}$ photoanode studied in this work (Supplementary Fig. 1c). As shown in the DFT results, the terminal and middle hydroxyl groups of glycerol adsorb spontaneously on the bismuth site of $\mathrm{BiVO}_{4}$ with strong electrostatic attraction between $\mathrm{Bi}^{3+}$ and oxygen of hydroxyls (Fig. 5, Supplementary Fig. 20, and Supplementary Note 11). Subsequently, the adsorbed glycerol is oxidized by the photogenerated holes in $\mathrm{BiVO}_{4}$ to form radicals (Supplementary Figs. 21 and 22). The tertiary radical on the middle carbon is 0.18 $\mathrm{eV}$ more stable than the terminal radical, which explains the good selectivity towards DHA production during photoelectrochemical oxidation of glycerol on $\mathrm{BiVO}_{4}$. The photo-assisted reaction is critical for the production of DHA as only formic acid could be detected in the final liquid product under dark condition. $\mathrm{BiVO}_{4}$ provides a suitable bandgap of $\sim 2.5 \mathrm{eV}$ that endows photogenerated holes with sufficient energy to overcome the energy requirement for the formation of radicals, which is $\sim 1.5 \mathrm{eV}$. Thereafter, the radicals react with water, followed by dehydration to eventually form DHA.

Finally, incident photon-to-DHA quantum efficiency (QEDHA) was evaluated by multiplying IPCE with faradaic efficiency of DHA as shown in Fig. $4 \mathrm{f}$. In the reaction medium with $\mathrm{pH}=2$, a 25\% photon-to-DHA conversion efficiency can be achieved at a wavelength of $400 \mathrm{~nm}$.

\section{Discussion}

In summary, we have demonstrated an efficient and stable photoelectrochemical system based on porous $\mathrm{BiVO}_{4}$ nanoarrays for selective oxidation of glycerol to high value-added DHA. In an $\mathrm{H}-$ type cell at $\mathrm{pH}=2$, the porous $\mathrm{BiVO}_{4}$ nanoarray photoanode is able to produce $56 \mathrm{mmol} \mathrm{g}_{\text {catalyst }}{ }^{-1} \mathrm{~h}^{-1}$ of DHA at a potential of $1.2 \mathrm{~V}$ vs. RHE under AM 1.5 illumination $\left(100 \mathrm{~mW} \mathrm{~cm}^{-2}\right)$. Based on isotope labeling and DFT calculations, we deduced a possible reaction mechanism of selective photoelectrochemical oxidation of glycerol to DHA on $\mathrm{BiVO}_{4}$. This study not only provides an evidence of the reaction process of selective photoelectrochemical oxidation of glycerol to DHA, but also presents a broad research a

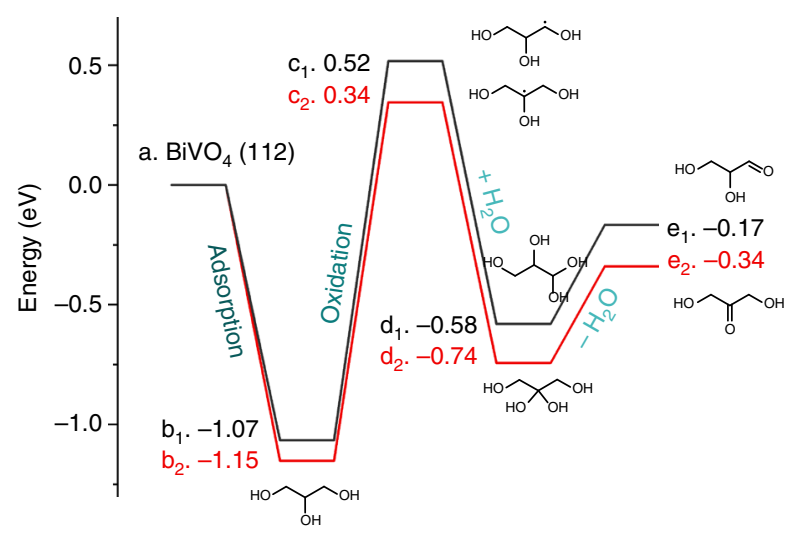

b

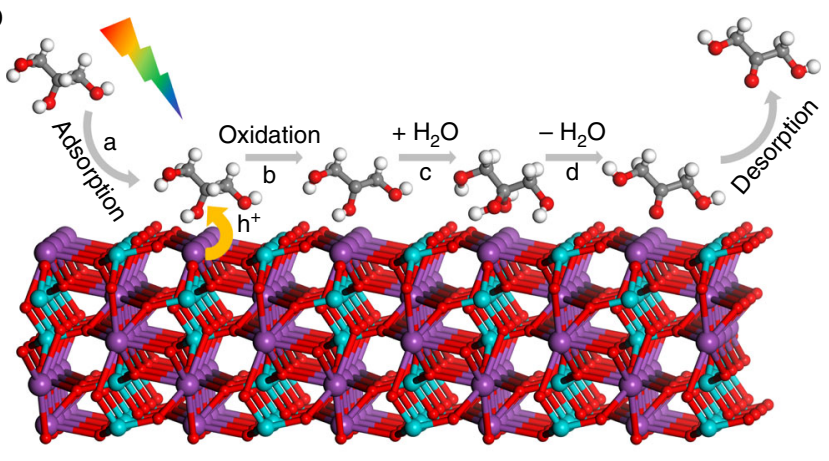

-Bi $\odot \mathrm{V} \odot \mathrm{O} \odot \mathrm{CH}$

Fig. 5 Reaction mechanism. a Energy profile of glycerol oxidation on $\mathrm{BiVO}_{4}$. 1 (black) and 2 (red) stand for reactions that take place at the terminal and middle carbon, respectively. b Schematic illustration showing PEC glycerol oxidation to DHA

perspective for the utilization of solar light to produce high valueadded chemicals from renewable feedstocks.

\section{Methods}

Synthesis of $\mathbf{B i V O}_{4}$ photoanode. All chemicals were purchased from SigmaAldrich and used without further purification. $\mathrm{BiVO}_{4}$ photoanode was prepared by reacting $\mathrm{BiOI}$ nanoflake arrays with vanadyl acetylacetonate ${ }^{38}$. Firstly, BiOI nanoflake arrays were grown on conductive fluorine-doped $\mathrm{SnO}_{2}(\mathrm{FTO}, 14 \Omega \mathrm{cm}$, $1 \times 1 \mathrm{~cm}^{2}$ ) by a three-electrode cathodic electrodeposition at $-0.1 \mathrm{~V}$ vs. $\mathrm{Ag} / \mathrm{AgCl}$ for $5 \mathrm{~min}$ in a mixed solution of $50 \mathrm{~mL}$ of $0.04 \mathrm{M} \mathrm{Bi}\left(\mathrm{NO}_{3}\right)_{3}$ and $0.4 \mathrm{M} \mathrm{KI}$ aqueous solution with $\mathrm{pH}$ adjusted to 1.7 by adding $\mathrm{HNO}_{3}$ and $20 \mathrm{~mL}$ of $0.23 \mathrm{M}$ benzoquinone ethanol solution. Subsequently, $0.1 \mathrm{~mL}$ of vanadyl acetylacetonate dimethyl sulfoxide solution $(0.4 \mathrm{M})$ was added onto the BiOI nanoflake arrays, followed by heating to $723 \mathrm{~K}$ in ambient environment at a ramp rate of $2 \mathrm{~K} \mathrm{~min}^{-1}$. After $2 \mathrm{~h}$, the resultant product on FTO was cooled to room temperature and washed by $\mathrm{NaOH}$ solution.

Sample characterizations. SEM images were taken on a JEOL JSM-6700F field emission scanning electron microscope operated at $5 \mathrm{kV}$. HRTEM image was taken on a JEOL JEM-2100F field-emission transmission electron microscope operated at $200 \mathrm{kV}$. X-ray powder diffraction (XRD) patterns were recorded using a Philips X'Pert Pro Super X-ray diffractometer with $\mathrm{Cu} \mathrm{Ka}$ radiation $(\lambda=1.54178 \AA$ ). $\mathrm{UV}$-vis diffuse reflectance spectrum was recorded in the spectral region of $300-600 \mathrm{~nm}$ on a Shimadzu SolidSpec-3700 spectrophotometer. The bandgap of $\mathrm{BiVO}_{4}$ was determined based on the Tauc plot. $\mathrm{BiVO}_{4}$ photocatalysts used in this work were scraped from the $\mathrm{BiVO}_{4}$ nanoarray-FTO glass.

Fourier transform infrared spectroscopy (FTIR) measurements were carried out in the attenuated total reflection (ATR) configuration using a Nicolet iN10 MX spectrometer equipped with a Ge ATR crystal and a DTGS KBr detector. All spectra were measured with a resolution of $1 \mathrm{~cm}^{-1}$ and presented in absorbance $A=-\log \left(R / R_{\mathrm{o}}\right)$, where $R$ and $R_{\mathrm{o}}$ are the reflectance spectra of $\mathrm{BiVO}_{4}$ samples treated in electrolyte of different $\mathrm{pH}$ with and without glycerol. After soaked in the electrolyte with and without glycerol at different $\mathrm{pH}$ for $10 \mathrm{~min}$, the $\mathrm{BiVO}_{4}$ samples were washed by the electrolyte without glycerol. After dried with $\mathrm{N}_{2}$ flow, the $\mathrm{BiVO}_{4}$ samples were pressed into intimate optical contact with the Ge crystal for ATR-FTIR measurements. 
Room-temperature ESR spectra of $\mathrm{BiVO}_{4}$ photocatalysts were collected on a JEOL JES-FA200 ESR spectrometer $(300 \mathrm{~K}, 9.063 \mathrm{GHz}$, X-band). Microwave power employed was $1 \mathrm{~mW}$ and sweep width was set from 319.3 to $329.3 \mathrm{mT}$. Modulation frequency and modulation amplitude were $100 \mathrm{kHz}$ and $0.35 \mathrm{mT}$, respectively. 5,5Dimethyl-1-pyrroline $\mathrm{N}$-oxide (DMPO) was used as the spin-trapping agent. $0.5 \mathrm{mg} \mathrm{BiVO}$ photocatalysts were dispersed in $2 \mathrm{~mL} 0.5 \mathrm{M} \mathrm{Na}_{2} \mathrm{SO}_{4}$ electrolyte $(\mathrm{pH}=2)$ with or without $0.1 \mathrm{M}$ glycerol, then $60 \mu \mathrm{L}$ DMPO aqueous solution $(0.2 \mathrm{M})$ was added into the solution for ESR measurement.

Photoelectrochemical characterization. Photoelectrochemical measurements were conducted on an electrochemical workstation (CHI 760E) in a sealed H-type glass cell. Nafion 212 proton exchange membrane was used to separate the anode from the cathode chamber. The simulated solar illumination was obtained from a $300 \mathrm{~W}$ Xenon lamp with an AM 1.5G filter $\left(100 \mathrm{~mW} \mathrm{~cm}^{-2}\right)$. The porous $\mathrm{BiVO}_{4}$ nanoarrays grown on FTO were front illuminated and used as the working electrode, while $\mathrm{Ag} / \mathrm{AgCl}$ electrode and $\mathrm{Pt}$ sheet were used as the reference and counter electrode, respectively. The electrolyte contains $0.5 \mathrm{M} \mathrm{Na}_{2} \mathrm{SO}_{4}$ aqueous solution with $\mathrm{pH}$ adjusted to $2,5,7,10$, and 12 using $1 \mathrm{M} \mathrm{NaOH}$ solution or $0.5 \mathrm{M} \mathrm{H}_{2} \mathrm{SO}_{4}$ solution. Photocurrent was recorded from 0.2 to $1.4 \mathrm{~V}$ vs. RHE at a scan rate of $10 \mathrm{mV} \mathrm{s}^{-1}$ with and without the presence of $0.1 \mathrm{M}$ glycerol.

The photoresponse of the $\mathrm{BiVO}_{4}$ photoanode was studied by measuring photocurrent densities under chopped light irradiation (light on/off cycles: $20 \mathrm{~s}$ ) at 0.6 and $1.0 \mathrm{~V}$ vs. $\mathrm{RHE}$ in $0.5 \mathrm{M} \mathrm{Na}_{2} \mathrm{SO}_{4}$ at various $\mathrm{pH}$ with and without glycerol. The photoelectrochemical impedance spectra were collected in a frequency range of $1 \sim 10^{5} \mathrm{~Hz}$ with an amplitude of $5 \mathrm{mV}$ under AM $1.5 \mathrm{G}, 100 \mathrm{~mW} \mathrm{~cm}{ }^{-2}$ illumination, from $0.6 \mathrm{~V}$ to $1.4 \mathrm{~V}$ vs. RHE. Mott-Schottky plots were measured at $1000 \mathrm{~Hz}$ under dark and AM $1.5 \mathrm{G}, 100 \mathrm{~mW} \mathrm{~cm}^{-2}$ illumination with potential varied from -0.3 to $0.6 \mathrm{~V}$ vs. RHE.

OCVD measurements were carried out with light illumination on and off. After turning off the light, the average lifetime of photogenerated charge carriers $\left(\tau_{n}\right)$ was estimated according to:

$$
\tau_{\mathrm{n}}=\frac{k_{\mathrm{B}} T}{e}\left(\frac{\mathrm{d} V_{\mathrm{oc}}}{\mathrm{d} t}\right)^{-1}
$$

where $\tau_{\mathrm{n}}$ is the potential dependent lifetime, $k_{\mathrm{B}}$ is Boltzmann's constant, $T$ is the temperature in $\mathrm{K}, e$ is the single electron charge, and $V_{\mathrm{oc}}$ is the open-circuit voltage at time $t$.

IPCE was measured under monochromatic irradiation under one sun illumination (AM $1.5 \mathrm{G}, 100 \mathrm{~mW} \mathrm{~cm}^{-2}$ ) equipped with a monochromator at $1.23 \mathrm{~V}$ vs. RHE:

$$
\mathrm{IPCE}=\frac{\left[\left(\frac{1240}{\lambda}\right) \times\left(J_{\text {light }}-J_{\text {dark }}\right)\right]}{P} \times 100 \%
$$

where $\lambda$ is the wavelength, $J_{\text {light }}$ is the photocurrent density under irradiation, $J_{\text {dark }}$ is the current density under dark condition, and $P$ is the incident light power density.

Photoelectrocatalysis characterizations. To quantitatively analyze the oxidation products, the photoelectrochemical oxidation was performed in a sealed $\mathrm{H}$-type cell at a potential of $0.6,0.8,1.0$, and $1.2 \mathrm{~V}$ vs. RHE for $1 \mathrm{~h}$ without the addition of adscititious oxidant. After reaction, $1 \mathrm{~mL}$ of solution was taken out from the cell and analyzed using high performance liquid chromatography (HPLC) to calculate the glycerol conversion and yields of the products using an Agilent 1260 chromatograph equipped with a Hi-Plex H $(300 \times \mathrm{d}-7 \mathrm{~mm}, 8 \mu \mathrm{m})$ column and MWD and RID detector. The external quantum efficiency of photon-to-DHA was calculated based on IPCE and faradaic efficiency of DHA.

Selectivity of DHA was calculated based on:

$$
\begin{aligned}
\text { Selectivity }(\mathrm{DHA})=\frac{n_{\mathrm{DHA}}}{n_{\mathrm{all}}} \times 100 \% & =\frac{n_{\mathrm{DHA}}}{n_{\mathrm{GA}}+n_{\mathrm{DHA}}+n_{\mathrm{FA}}+n_{\mathrm{GlA}}+n_{\mathrm{LA}}} \times 100 \% \\
& =\frac{c_{\mathrm{DHA}}}{c_{\mathrm{GA}}+c_{\mathrm{DHA}}+c_{\mathrm{FA}}+c_{\mathrm{GlA}}+c_{\mathrm{LA}}} \times 100 \%
\end{aligned}
$$

where $n_{\mathrm{DHA}}, n_{\mathrm{GA}}, n_{\mathrm{FA}}, n_{\mathrm{GlA}}$, and $n_{\mathrm{LA}}$ are the yield of DHA, glyceric acid, formic acid, glycolic acid, and lactic acid, respectively. $c$ is the product concentration detected by HPLC.

Production rate of DHA was calculated according to:

$$
\begin{gathered}
\text { Production rate }(\mathrm{DHA})=\frac{c_{\mathrm{DHA}} \times V}{t} \\
\text { Production rate per unit area }(\mathrm{DHA})=\frac{c_{\mathrm{DHA}} \times V}{t \times A}
\end{gathered}
$$

where $V$ is the volume of reaction solution, $t$ is the reaction time, and $A$ is the area of photoanode.
Faradaic efficiency was calculated by:

$$
\begin{aligned}
\text { Faradaic efficiency }(\mathrm{DHA}) & =\frac{\text { Number of holes to oxidize glycerol to DHA }}{\text { Number of all collected photogenerated holes }} \times 100 \% \\
& =\frac{e_{\mathrm{DHA}} \times n_{\mathrm{DHA}} \times N}{\mathrm{Q} / n} \times 100 \% \\
& =\frac{2 \times c_{\mathrm{DHA}} \times V \times N}{\mathrm{Q} / n} \times 100 \%
\end{aligned}
$$

where $e_{\mathrm{DHA}}$ is the number of holes required to oxidize one glycerol molecule to DHA, $N$ is Avogadro's constant, $Q$ is the quantity of electric charge, and $n$ is the elementary charge.

External quantum efficiency of DHA was calculated by:

$$
\begin{aligned}
\mathrm{QE}(\mathrm{DHA}) & =\frac{\text { Number of holes to oxidize glycerol to DHA }}{\text { Number of incident photons }} \times 100 \% \\
& =\mathrm{IPCE} \times \text { Faradaic efficiency }(\mathrm{DHA}) \times 100 \%
\end{aligned}
$$

Carbon balance was calculated based on:

$$
\text { Carbon balance }=\frac{n_{\mathrm{GA}}+n_{\mathrm{DHA}}+\frac{n_{\mathrm{FA}}}{3}+\frac{n_{\mathrm{Cl}}}{3 / 2}+\frac{n_{\mathrm{CO} 2}}{3}+\frac{n_{\mathrm{CO}}}{3}}{n_{\text {glycerol }}} \times 100 \%
$$

The LC-MS measurements were carried out on a LTQ Orbitrap XL Hybrid Ion Trap-Orbitrap Mass Spectrometer with IonMax ESI (electrospray ionization) Source. $0.5 \mathrm{M}$ of $\mathrm{Na}_{2} \mathrm{SO}_{4}$ solution containing $10 \% \mathrm{H}_{2}{ }^{18} \mathrm{O}$ was used as the electrolyte with $\mathrm{pH}$ adjusted to 2 by $0.5 \mathrm{M}$ of $\mathrm{H}_{2} \mathrm{SO}_{4}$. After $2 \mathrm{~h}$ of PEC reaction, the products with electrolyte were collected. Before LC-MS measurements, the products were extracted by SPE (Solid Phase Extraction Cartridges) column to remove inorganic salts. Hypersil GOLD column ( $3 \mu \mathrm{m}$ particle size) and ESI $\mathrm{Na}^{+}$source were used in the LC-MS measurements.

Computational details. All DFT calculations were performed with VASP 5.3.5 $5^{39,40}$. Spin-polarized DFT with Perdew-Burke-Ernzerhof (PBE) flavor of generalized gradient approximation were employed ${ }^{41}$. Iterative solutions of the Kohn-Sham equations were expanded in plane-wave basis sets defined by a kinetic energy cutoff of $400 \mathrm{eV}$. The convergences of energy and force were set as $10^{-6} \mathrm{eV}$ and $0.02 \mathrm{eV}^{-1}$, respectively. Single gamma point sampling was used for the Brillouin Zone integration. The $\mathrm{BiVO}_{4}(112)$ surface was modeled by a $\mathrm{p}(2 \times 2)$ supercell with three $\mathrm{VO}_{4}$ layers as shown in Supplementary Fig. 20a. The calculated interlayer distance is $3.10 \AA$, which is in good agreement with HRTEM results. A vacuum region of $15 \AA$ was set between the periodically repeated slabs to avoid artificial interactions. The charge density differences were evaluated using the formula $\Delta \rho=\rho_{\text {slab }+ \text { ads }}-\rho_{\text {slab }}-\rho_{\text {ads }}$, where $\rho_{\text {slab }}, \rho_{\text {ads }}$, and $\rho_{\text {slab }+ \text { ads }}$ are the electron densities of $\mathrm{BiVO}_{4}(112)$ slab, adsorbate, and the adsorption configuration, respectively.

\section{Data availability}

All data supporting the findings in the article as well as the Supplementary Information files are available from the corresponding authors on reasonable request. The source data underlying Fig. 1 and Supplementary Figs. 5, 10a-c, and 18 are provided as a Source Data file.

Received: 14 July 2018 Accepted: 22 March 2019

Published online: 16 April 2019

\section{References}

1. Bender, T. A., Dabrowski, J. A. \& Gagné, M. R. Homogeneous catalysis for the production of low-volume, high-value chemicals from biomass. Nat. Rev. Chem. 2, 35-46 (2018).

2. Lewis, N. S. \& Nocera, D. G. Powering the planet: chemical challenges in solar energy utilization. Proc. Natl. Acad. Sci. USA 103, 15729-15735 (2006).

3. Li, W. et al. Integrated photoelectrochemical solar energy conversion and organic redox flow battery devices. Angew. Chem. Int. Ed. 55, 13104-13108 (2016).

4. Nocera, D. G. Solar fuels and solar chemicals industry. Acc. Chem. Res. 50, 616-619 (2017).

5. Caes, B. R., Teixeira, R. E., Knapp, K. G. \& Raines, R. T. Biomass to furanics: renewable routes to chemicals and fuels. ACS Sustain. Chem. Eng. 3, 2591-2605 (2015)

6. Caspeta, L., Buijs, N. A. A. \& Nielsen, J. The role of biofuels in the future energy supply. Energy Environ. Sci. 6, 1077-1082 (2013).

7. Issariyakul, T. \& Dalai, A. K. Biodiesel from vegetable oils. Renew. Sustain. Energy Rev. 31, 446-471 (2014).

8. Knothe, G., Van Gerpen, J. H. \& Krahl, J. The Biodiesel Handbook (AOCS Press, Champaign, Illinois, USA, 2005).

9. Lee, A. F., Bennett, J. A., Manayil, J. C. \& Wilson, K. Heterogeneous catalysis for sustainable biodiesel production via esterification and transesterification. Chem. Soc. Rev. 43, 7887-7916 (2014). 
10. Pradhan, A. et al. Energy life-cycle assessment of soybean biodiesel revisited. Trans. ASABE 54, 1031-1039 (2011).

11. Haider, M. H. et al. Efficient green methanol synthesis from glycerol. Nat. Chem. 7, 1028-1032 (2015).

12. Morales, M. et al. Environmental and economic assessment of lactic acid production from glycerol using cascade bio- and chemocatalysis. Energy Environ. Sci. 8, 558-567 (2015).

13. Bagheri, S., Julkapli, N. M. \& Yehye, W. A. Catalytic conversion of biodiesel derived raw glycerol to value added products. Renew. Sustain. Energy Rev. 41, 113-127 (2015).

14. Pagliaro, M., Ciriminna, R., Kimura, H., Rossi, M. \& Della Pina, C. From glycerol to value-added products. Angew. Chem. Int. Ed. 46, 4434-4440 (2007).

15. Katryniok, B. et al. Selective catalytic oxidation of glycerol: perspectives for high value chemicals. Green Chem. 13, 1960-1979 (2011).

16. Lam, C. H., Bloomfield, A. J. \& Anastas, P. T. A switchable route to valuable commodity chemicals from glycerol via electrocatalytic oxidation with an earth abundant metal oxidation catalyst. Green Chem. 19, 1958-1968 (2017).

17. Zalineeva, A. et al. Self-supported $\mathrm{Pd}_{x} \mathrm{Bi}$ catalysts for the electrooxidation of glycerol in alkaline media. J. Am. Chem. Soc. 136, 3937-3945 (2014).

18. Kumar, G. S. et al. Stabilized glycerol dehydrogenase for the conversion of glycerol to dihydroxyacetone. Chem. Eng. J. 276, 283-288 (2015).

19. Quispe, C. A. G., Coronado, C. J. R. \& Carvalho, J. A. Glycerol: production, consumption, prices, characterization and new trends in combustion. Renew. Sustain. Energy Rev. 27, 475-493 (2013).

20. Ma, L., Lu, W., Xia, Z. \& Wen, J. Enhancement of dihydroxyacetone production by a mutant of Gluconobacter oxydans. Biochem. Eng. J. 49, 61-67 (2010).

21. Ciriminna, R., Palmisano, G., Della Pina, C., Rossi, M. \& Pagliaro, M. One-pot electrocatalytic oxidation of glycerol to DHA. Tetrahedron Lett. 47, 6993-6995 (2006).

22. Ning, X. et al. Promoting role of bismuth and antimony on Pt catalysts for the selective oxidation of glycerol to dihydroxyacetone. J. Catal. 335, 95-104 (2016).

23. Kwon, Y., Birdja, Y., Spanos, I., Rodriguez, P. \& Koper, M. T. M. Highly selective electro-oxidation of glycerol to dihydroxyacetone on platinum in the presence of bismuth. ACS Catal. 2, 759-764 (2012).

24. Schunemann, S., Dodekatos, G. \& Tüysüz, H. Mesoporous silica supported Au and $\mathrm{AuCu}$ nanoparticles for surface plasmon driven glycerol oxidation. Chem. Mater. 27, 7743-7750 (2015).

25. Ribeiro, L. S. et al. Pd, Pt, and Pt-Cu catalysts supported on carbon nanotube (CNT) for the selective oxidation of glycerol in alkaline and base-free conditions. Ind. Eng. Chem. Res. 55, 8548-8556 (2016).

26. Hirasawa, S., Watanabe, H., Kizuka, T., Nakagawa, Y. \& Tomishige, K. Performance, structure and mechanism of $\mathrm{Pd}-\mathrm{Ag}$ alloy catalyst for selective oxidation of glycerol to dihydroxyacetone. J. Catal. 300, 205-216 (2013).

27. Garcia, A. C. et al. Strong impact of platinum surface structure on primary and secondary alcohol oxidation during electro-oxidation of glycerol. ACS Catal. 6, 4491-4500 (2016)

28. Garcia, A. C., Birdja, Y. Y., Tremiliosi-Filho, G. \& Koper, M. T. M. Glycerol electro-oxidation on bismuth-modified platinum single crystals. J. Catal. 346, 117-124 (2017).

29. Dotan, H., Sivula, K., Gratzel, M., Rothschild, A. \& Warren, S. C. Probing the photoelectrochemical properties of hematite $\left(\alpha-\mathrm{Fe}_{2} \mathrm{O}_{3}\right)$ electrodes using hydrogen peroxide as a hole scavenger. Energy Environ. Sci. 4, 958-964 (2011).

30. Klahr, B., Gimenez, S., Fabregat-Santiago, F., Bisquert, J. \& Hamann, T. W. Electrochemical and photoelectrochemical investigation of water oxidation with hematite electrodes. Energy Environ. Sci. 5, 7626-7636 (2012).

31. Klahr, B., Gimenez, S., Zandi, O., Fabregat-Santiago, F. \& Hamann, T. Competitive photoelectrochemical methanol and water oxidation with hematite electrodes. ACS Appl. Mater. Interfaces 7, 7653-7660 (2015).

32. Heo, N., Jun, Y. \& Park, J. H. Dye molecules in electrolytes: new approach for suppression of dye-desorption in dye-sensitized solar cells. Sci. Rep. 3, 1712 (2013).

33. Palmisano, G. et al. Advances in selective conversions by heterogeneous photocatalysis. Chem. Commun. 46, 7074-7089 (2010).

34. Kongjao, S., Damronglerd, S. \& Hunsom, M. Electrochemical reforming of an acidic aqueous glycerol solution on Pt electrodes. J. Appl. Electrochem. 41, 215-222 (2011).
35. Zhang, M. et al. Oxygen atom transfer in the photocatalytic oxidation of alcohols by $\mathrm{TiO}_{2}$ : oxygen isotope studies. Angew. Chem. Int. Ed. 48, 6081-6084 (2009)

36. Vaz, A. D. N. \& Coon, M. J. On the mechanism of action of cytochrome P450: evaluation of hydrogen abstraction in oxygen-dependent alcohol oxidation. Biochemistry 33, 6442-6449 (1994).

37. Hu, W., Knight, D., Lowry, B. \& Varma, A. Selective oxidation of glycerol to dihydroxyacetone over $\mathrm{Pt}-\mathrm{Bi} / \mathrm{C}$ catalyst: optimization of catalyst and reaction conditions. Ind. Eng. Chem. Res. 49, 10876-10882 (2010).

38. Kim, T. W. \& Choi, K.-S. Nanoporous $\mathrm{BiVO}_{4}$ photoanodes with dual-layer oxygen evolution catalysts for solar water splitting. Science 343, 990-994 (2014).

39. Kresse, G. \& Furthmüller, J. Efficiency of ab-initio total energy calculations for metals and semiconductors using a plane-wave basis set. Comput. Mater. Sci. 6, 15-50 (1996).

40. Kresse, G. \& Furthmüller, J. Efficient iterative schemes for ab initio totalenergy calculations using a plane-wave basis set. Phys. Rev. B 54, 11169-11186 (1996).

41. Perdew, J. P., Burke, K. \& Ernzerhof, M. Generalized gradient approximation made simple. Phys. Rev. Lett. 77, 3865-3868 (1996).

\section{Acknowledgements}

This work was supported by the funds from the Singapore Ministry of Education Academic Research Fund (AcRF) Tier 1: RG115/18, RG115/17 and RG9/17, Tier 2: MOE2016-T2-2-004, the National Key Projects for Fundamental Research and Development of China (2017YFA0207301, 2016YFA0202804), the Strategic Priority Research Programme of the Chinese Academy of Sciences (XDB17020400), and the National Nature Science Foundation of China (21725102).

\section{Author contributions}

D.L. and B.L. conceived the project and designed the experiments. B.L. supervised the project. D.L. carried out experiments and data analysis. J.-C.L., H.X., and J.L. performed numerical calculation. W.C., J.M., and H.B.Y. contributed to catalyst characterization. Y. X. and Y.H. contributed to the manuscript preparation. D.L. and B.L. together wrote the manuscript. All authors commented on the final manuscript.

\section{Additional information}

Supplementary Information accompanies this paper at https://doi.org/10.1038/s41467019-09788-5.

Competing interests: The authors declare no competing interests.

Reprints and permission information is available online at http://npg.nature.com/ reprintsandpermissions/

Journal peer review information: Nature Communications thanks the anonymous reviewers for their contribution to the peer review of this work. Peer reviewer reports are available.

Publisher's note: Springer Nature remains neutral with regard to jurisdictional claims in published maps and institutional affiliations.

\footnotetext{
Open Access This article is licensed under a Creative Commons Attribution 4.0 International License, which permits use, sharing, adaptation, distribution and reproduction in any medium or format, as long as you give appropriate credit to the original author(s) and the source, provide a link to the Creative Commons license, and indicate if changes were made. The images or other third party material in this article are included in the article's Creative Commons license, unless indicated otherwise in a credit line to the material. If material is not included in the article's Creative Commons license and your intended use is not permitted by statutory regulation or exceeds the permitted use, you will need to obtain permission directly from the copyright holder. To view a copy of this license, visit http://creativecommons.org/ licenses/by/4.0/
}

(C) The Author(s) 2019 